\title{
Modeling of positive and negative organic magnetoresistance in organic light-emitting diodes
}

\author{
Sijie Zhang, N. J. Rolfe, P. Desai, P. Shakya, A. J. Drew, T. Kreouzis, and W. P. Gillin \\ School of Physics and Astronomy, Queen Mary University of London, Mile End Road, London, E1 4NS, United Kingdom
}

(Received 17 May 2011; revised manuscript received 30 July 2012; published 17 August 2012)

\begin{abstract}
The organic magnetoresistance of aluminium tris(8-hydroxyquinoline) based organic light-emitting diodes has been modeled using the triplet polaron interaction coupled with exciton dissociation. We have demonstrated that each of the processes is proportional to the exciton concentration over a wide range of operating conditions for a number of devices with a wide range of layer thicknesses. This work demonstrates that using a magnetic field to perturb the operation of a working organic device is particularly valuable in that it provides a new technique for studying a range of processes affecting current transport such as polaron trapping, triplet-polaron interactions, and exciton dissociation in fully working devices.
\end{abstract}

DOI: 10.1103/PhysRevB.86.075206

PACS number(s): 72.80.Le, 73.61.Ph, 73.43.Qt

\section{INTRODUCTION}

The effect of magnetic fields on a variety of spin-dependent processes in organic materials has been researched since the 1960s. ${ }^{1-3}$ However, it is only in recent years that there has been a dramatic increase in the numbers of papers investigating the coupling of these interactions of molecules with magnetic fields and their effect on macroscopic processes, such as the current transfer through devices. Initial work focused on studying fundamental interactions such as triplet quenching, ${ }^{1}$ triplet-triplet annihilation, ${ }^{2}$ and intersystem crossing (ISC). ${ }^{3}$ These studies were predominantly performed on molecular crystal or solutions, however, despite the advent of efficient organic light-emitting devices (OLEDs) in $1986,{ }^{4}$ it took nearly 20 years before the dramatic effects of a magnetic field on working OLEDs was investigated. Kalinowski et al. ${ }^{5}$ were the first to observe that the photoconductivity of an organic device could be perturbed by the presence of a magnetic field and they then went on to demonstrate that magnetic fields could significantly alter the current through an OLED, now referred to as organic magnetoresistance (OMR or OMAR), as well as its light output. ${ }^{6}$ The term magnetoresistance is not strictly correct as the resistance of an OLED is highly nonlinear with drive voltage. However, the phrase is now widely used so we will keep with that nomenclature despite the fact that, like most authors, we use it to refer to a change in the current in the device at a constant drive voltage. Since Kalinowski's work the study of these phenomena has increased dramatically ${ }^{7-43}$ although there is still not complete consensus between the fundamental interactions that have been observed to occur on a molecular scale and the macroscopic effects such as a change in current and device efficiency.

In 2007, we suggested that the change in current with applied magnetic field could be due to changes in triplet exciton population through ISC at either the excitonic or pair level interactions. ${ }^{15}$ Pair level interactions have been proposed by a number of groups and describe how the spins of polarons can be flipped prior to exciton formation and they are generally believed to occur due to hyperfine interactions. ${ }^{6,13}$ These interactions are highly similar to the processes that are suggested for the bipolaron model of OMR, ${ }^{35}$ although in that case the interactions are between polarons of the same charge. Mixing between singlets and triplets once the exciton has been formed (ISC) is well known and is generally assumed to have a significant influence from spin-orbit interactions. Transitions from a triplet to singlet state can occur if the triplet is raised in to a higher excited state, which can occur due to excited state absorption. ${ }^{44}$ These changes in triplet population could then affect the current through a device via a number of processes that affect the mobility, for example, the role of weak electrostatic trapping of polarons at triplets or spin blocking; the role of excitons as shallow traps was theoretically proposed by Agranovich et al., ${ }^{45}$ and the effect of excitons on mobility has recently been experimentally verified using dark injection experiments. ${ }^{46,47}$ Our initial work focused on the role of site blocking and polaron interactions with excitons, which we suggested as a cause for the increase in current through a device with magnetic field. We have recently shown that the OMR can be modeled using just two processes, ${ }^{39}$ site blocking or weak electrostatic trapping of polarons with triplets, as proposed by Agranovich, ${ }^{45}$ and interactions of polarons and triplet in strongly coupled states, similar to that observed by Ern and Merrifield. ${ }^{1}$

Throughout our previous works we have not precisely defined the nature of the interaction between triplets and polarons as there are a number of possible reactions that may occur that could affect the device current. Furthermore, it is not unreasonable to assume that in real devices some or all of these processes may be occurring in parallel. Examples of triplet polaron interactions could include: quenching of the triplet by the polaron, ${ }^{1,48}$ dissociation of the coupled state back into a polaron and triplet (which is effectively a scattering event ${ }^{1}$ ) and dissociation of the triplet by the polaron to produce two new polarons. The first two of these processes are known to be spin dependent ${ }^{1,48}$ and would therefore be expected to have a magnetic field dependence. However, even the spin independent triplet dissociation process will probably be occurring in parallel with the other processes and hence the balance between the different processes would be expected to change if the rate of any one of them changed. Therefore even the spin independent process may have some magnetic field dependence due to changes in the other processes; for example, changing the quenching rate will affect the triplet population and this may have an effect on the amount of dissociation occurring. It is therefore difficult to determine precisely which effect results in a change in device current, which is why we have, so far, grouped them together as generic triplet-polaron interactions. Despite this caution, there is ample 
experimental evidence that these processes can occur although experiments designed to detect individual interactions are not necessarily directly coupled to current changes in the presence of a magnetic field. For example, the quenching of triplet by polarons was observed by Ern and Merrifield and has since been also been observed using photoluminescence detected magnetic resonance (PLDMR) ${ }^{48}$ in organic semiconductors. In order to fully unravel the precise effects responsible for OMR, further work will be needed to couple OMR measurements with spin-sensitive probes such as electron paramagnetic resonance techniques. Much work has been done using such probes and they will be vital to obtain a full understanding of OMR. ${ }^{48-54}$

We have previously shown that for thin aluminium tris(8hydroxyquinoline) $\left(\mathrm{Alq}_{3}\right)$ based devices both positive and negative changes in device current could be obtained and the negative changes in current were attributed to the effect of exciton dissociation at the cathode ${ }^{16}$ and that the negative OMR could be removed if the excitons were prevented from reaching the cathode by the introduction of an exciton blocking layer. In this paper, we extend our model of positive OMR to include the effect of exciton dissociation at the cathode and demonstrate that for all our $\mathrm{Alq}_{3}$ devices, of any thickness, the OMR can be modeled using just three processes: spin blocking or trapping of polarons by triplets, spin-dependent interactions between polarons and triplets and triplet dissociation at the cathode. However, it must be stressed that even within these three processes there may be a greater number of interactions occurring that are hidden within a single term. For example, the effect of spin blocking and dissociation on the OMR is actually governed by changes in the triplet population and they may occur through both pair level and excitons level interactions. ${ }^{44}$

\section{EXPERIMENTAL METHOD}

OLED devices consisted of a patterned indium tin oxide (ITO) coated glass substrate on to which was grown a 50-nm layer of TPD as the hole transport layer, followed by an 11.5-, 15-, 20-, or 30-nm thick layer of $\mathrm{Alq}_{3}$ as an electron transport/emissive layer and $\operatorname{LiF}(1 \mathrm{~nm}) / \mathrm{Al}(100 \mathrm{~nm})$ cathode. All organic materials were purified by vacuum sublimation prior to use. Final device areas were $\sim 4 \mathrm{~mm}^{2}$. The ITO substrates were patterned using photolithography and cleaned by ultrasonicating in detergent/water, acetone, and chloroform. Following this, the ITO was treated in oxygen plasma for 5 minutes at $30 \mathrm{~W}$ and 2.5 mbar pressure using a Diener Electronic Femto Plasma system. The plasma treated substrate was immediately transferred to the deposition chamber for device fabrication. The deposition of the organic layers and metal electrodes were performed using a Kurt J. Lesker SPECTROS evaporation system with a base pressure during evaporation of $\sim 10^{-7}$ mbar. The rate of deposition of organic materials was $\sim 0.2 \mathrm{~nm} / \mathrm{s}$, while that of the aluminium was varied from $\sim 0.1$ to $0.5 \mathrm{~nm} / \mathrm{s}$. A calibrated oscillating quartz crystal monitor was used to determine the rate and thickness of the deposited layer. The whole device fabrication was performed without breaking vacuum.

Immediately after growth the devices were placed in a lighttight sample holder with a calibrated silicon photodetector (Newport 818-SL), whose output is independent of magnetic field, placed on the top surface of the device. The sample holder was placed between the poles of an electromagnet with the magnetic field perpendicular to the direction of current flow in the device. Thirty-two independent measurements were taken and then averaged with the device operated in vacuum and in constant voltage mode. Before and after each field measurement, a measurement at null field was taken and used to remove any effects due to drift in the device characteristics. The measurements were performed using a Keithley 236 source-measure unit and Newport 1830 optical power meter. All measurements were performed at room temperature.

\section{RESULTS AND DISCUSSION}

We have previously demonstrated that the positive OMR in $\mathrm{Alq}_{3}$ based devices could be modeled using two Lorentzians that corresponded to simple trapping or spin blocking of polarons at triplet excitons and interactions of strongly coupled triplet-polaron pairs. ${ }^{39}$ However, it is known that under certain circumstances it is possible to obtain negative OMR and it has been demonstrated that for $\mathrm{Alq}_{3}$ devices this negative OMR was only visible in thin devices and could be removed by the addition of an exciton blocking layer between the $\mathrm{Alq}_{3}$ layer and the cathode. ${ }^{16}$ It is well known that excitons can dissociate at an energetically favorable interface and the cathode is an ideal interface for dissociation as there are available states for both electrons and holes to couple to. In Fig. 1, we have illustrated the case for the hole in an exciton to tunnel into the cathode and the electric field in the device will then cause the electron to move back into the device. It is equally possible for the electron to tunnel into the cathode but in this situation the electric field will force the hole into the cathode too and the result will be the quenching of the triplet with no change in the
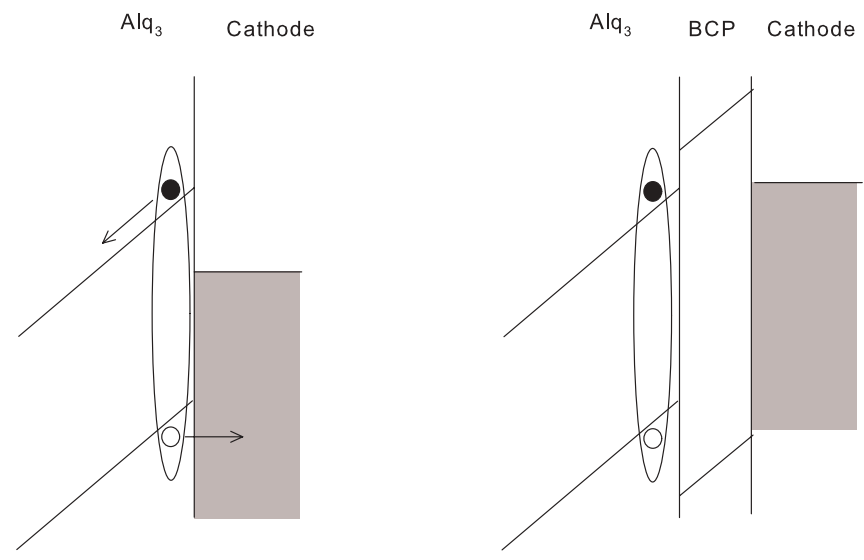

FIG. 1. A schematic diagram of an exciton at the interface between $\mathrm{Alq}_{3}$ and a cathode. Either the electron or hole can tunnel into the cathode as there are available states for either (i.e., an empty density of states above the Fermi level for electrons and a full density of states below the Fermi level for holes). If a hole tunnels out, then an electron is recycled back into the device, which would move away from the cathode by the electric field. If the electron were to tunnel out, then the electric field would result in the hole also entering the cathode so there would be no dissociation current. At the anode the situation would be reversed. The presence of an exciton blocking layer at this interface greatly reduced the probability of exciton dissociation. 
current in the device. Although we have only considered the interface with the cathode in this example, this dissociation can occur at any energetically favorable interface. At the $\mathrm{TPD} / \mathrm{Alq}_{3}$ interface where most of the excitons are expected to be generated, there is the possibility of exciton dissociation although here there is a significant energy barrier for both electrons and holes. Whilst the barrier for electrons from $\mathrm{Alq}_{3}$ to TPD is too high for transfer, the barrier for holes would be expected to be much lower. In that situation, the electric field would act to push the hole back into the $\mathrm{Alq}_{3}$ and where it may recombine with the surplus of electrons. Therefore given the poor energetic alignment any dissociation at this interface is expected to produce only a small contribution to the device current.

Due to these effects, there is therefore a high probability for excitons present at the cathode to dissociate with a corresponding recycling of electrons back into the device. In the steady state, the ratio of the triplet to singlet concentration should scale as the ratio of the lifetimes. Given that triplet excitons have a lifetime at least a thousand times longer than singlets, ${ }^{55-57}$ their concentration will be considerably higher in a working device and hence their dissociation would be expected to predominate. The change in efficiency of an OLED with applied magnetic field is a direct measure of the increase in singlet concentration per injected carrier. We have demonstrated that in OLEDs it is possible to obtain large changes in device efficiency with no change in the current present in the device. ${ }^{27}$ This is strongly suggestive that the change in efficiency is due to a change in the balance of singlet and triplet excitons due to interactions at both the pair state and excitonic level, which may be mediated through excited state absorption. ${ }^{43}$ Therefore an increase in efficiency can be taken as an increase in the singlet concentration which produces a corresponding reduction in the triplet concentration. ${ }^{15,39}$ As the triplet concentration at the interface is reduced, this means that the contribution to the device current produced by this dissociation will also be reduced. Therefore one would expect the reduction in the current due to a change in the triplet dissociation rate at the cathode, predicated by a change in the triplet concentration at that interface, to have the same magnetic field dependence as the measured change in efficiency.

The change in efficiency of an OLED with applied magnetic field at low drive voltages can be approximated by a single Lorentzian function, of the form $f(B) \sim B^{2} /\left(B^{2}+B_{0}^{2}\right)$, where $B$ is the applied magnetic field and $B_{0}$ is the saturation field. ${ }^{38}$ This function was first proposed by Mermer et al. ${ }^{10}$ and was shown by Sheng et al. to be a solution to the Hamiltonians for both hyperfine ${ }^{11}$ and spin-orbit ${ }^{20}$ interactions, and as such may be a generic expression for a spin interaction in the presence of a magnetic field in these systems. We have already demonstrated that in thick devices the OMR has a positive contribution from two processes which, depending on the relative spin states of the polaron and triplet, are due to the weak electrostatic trapping or spin blocking of polarons at triplets and the interaction between strongly interacting polarons and triplets. We have shown that these processes appear to be generic in $\mathrm{Alq}_{3}$ based devices and that their magnitude scales linearly with exciton concentration. Any negative contribution to the OMR will therefore have to be in addition to these processes. Given that we have shown that triplet dissociation at the cathode could be responsible for the negative OMR we have therefore had to add this component to our model. Any magnetic field effect on the dissociation component would be expected to scale as the triplet population at the interface and because the change in triplet population can be measured directly as the change in the efficiency of the device with magnetic field, and because this has already been shown to be Lorentzian in shape we have therefore fitted our data using a triple Lorentzian process of the form

$$
f(B)=a_{d} \frac{B^{2}}{\left(B^{2}+B_{d}^{2}\right)}+a_{t} \frac{B^{2}}{\left(B^{2}+B_{t}^{2}\right)}+a_{i} \frac{B^{2}}{\left(B^{2}+B_{i}^{2}\right)},
$$

where $B$ is the applied magnetic field, $a_{d}, \mathrm{a}_{t}$, and $a_{i}$ are the prefactors for the Lorentzians and $B_{d}, \mathrm{~B}_{t}$, and $B_{i}$ are the saturation fields, the subscripts $d, t$, and $i$ stand for dissociation, trapping and interaction respectively. As we have stated above, the magnetic field dependence of both the dissociation and trapping components should have the same functional form as the change in efficiency with applied field and hence $B_{d}$ should be equal to $B_{t}$. However, the dissociation gives a negative OMR (reducing the triplet concentration reduces dissociation and hence reduces current) whilst the effect of trapping is positive (reducing the triplet concentration reduces interactions between triplets and polarons and hence increase mobility), hence $a_{d}<0$ and $a_{t}>0$. Given these criteria it is not possible to explicitly differentiate between the two processes as, when fitting Eq. (1) to data, it is the difference between the two processes that determines the quality of the fit and $a_{d}$ and $a_{t}$ can have arbitrary values. For the values of the saturation fields, we have used the same approach that we used in our previous work ${ }^{38}$ and constrained the values to $5 \mathrm{mT}<B_{d}<7 \mathrm{mT}, 5 \mathrm{mT}<B_{t}<7 \mathrm{mT}$, and $B_{i}=160 \mathrm{mT}$.

Figure 2 shows the OMR curves for an 11.5-nm $\mathrm{Alq}_{3}$ device as a function of drive voltage with both double and triple Lorentzian fits. These thin devices show a negative OMR at low operating voltages, due to dissociation of triplets at the cathode, which can be removed by the inclusion of an exciton blocking layer between the $\mathrm{Alq}_{3}$ and the cathode. ${ }^{16}$ At low drive voltages, the double Lorentzian fit is indistinguishable from that of the triple Lorentzian supporting the view that the cause of the two low $B_{0}$ processes, dissociation and trapping, are both scaling with the change in triplet population with applied magnetic field. However, as the drive voltage is increased, it can be seen that there is an additional feature appearing at low magnetic fields where the OMR shows a small negative peak before rising rapidly. This feature can be fitted perfectly using the triple Lorentzian provided the saturation field for the dissociation and trapping component are allowed to vary slightly. The difference in the saturation field for the two components is not well defined due to the fact that the prefactors can be arbitrarily large. Therefore the standard error in the values of the saturation field for the two processes is of the order of 10-20\% and the difference between the two prefactors is always within one standard error of each other. However, despite the difficulty in obtaining precise values for the different saturation field, it is obvious that some difference is needed otherwise they would cancel each other out perfectly. 


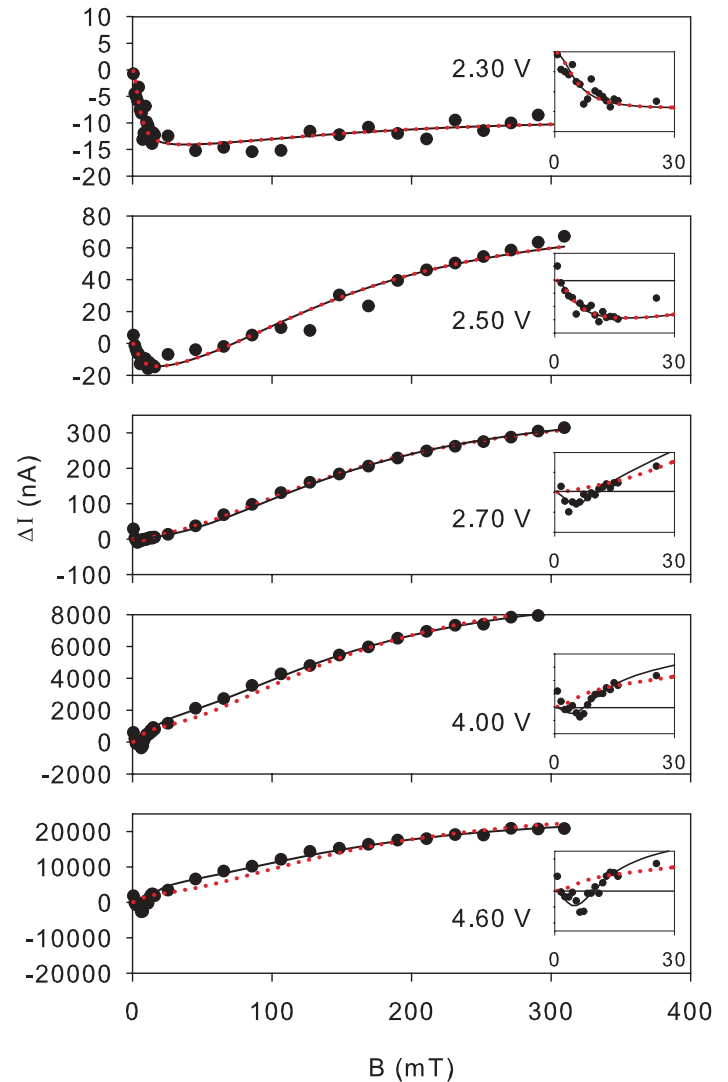

FIG. 2. (Color online) The absolute change in current for an 11.5-nm $\mathrm{Alq}_{3}$ OLED as a function of magnetic field for several different drive voltages. The solid line is the fit using the trapping, dissociation and interaction components [see Eq. (1)] and the dotted line is the fit using just the trapping and interaction terms. At low voltages, these two fits overlap perfectly. The inset for each graph is the detail of the low-field region from 0 to $30 \mathrm{mT}$.

It is therefore necessary to address why there should be any difference between them at all. We have stated that we believe that both the dissociation and trapping terms scale with the change in the triplet population in the device. The dissociation component is only governed by the triplet population at the interface between the $\mathrm{Alq}_{3}$ layer and the cathode. However, the trapping component is a bulk effect as any triplet in the layer can act as a trap and hence reduce the mobility. The small difference in the saturation fields therefore implies that the triplets near to the cathode are seeing a slightly different environment which is affecting the local ISC rate. This effect was also suggested by Wu et al..$^{58}$ who suggested that may be due to a change in the spin-orbit coupling induced by the metal cathode.

Identical behavior is seen for the 15-nm device (see Fig. 3), whereas once the thickness of the $\mathrm{Alq}_{3}$ layer is $20 \mathrm{~nm}$ (see Fig. 4) or greater, there is no evidence of the negative OMR at low voltages and the devices in this regime can be well modeled using just a dual Lorentzian. However, at drive voltages above $4 \mathrm{~V}$, the low-field component can no longer be fitted using just the trapping and interaction components and this suggests that the contribution from triplet dissociation is starting to become important. By $5 \mathrm{~V}$, there is a clear negative peak in the OMR data at $\sim 6 \mathrm{mT}$, which is, again, well fitted by
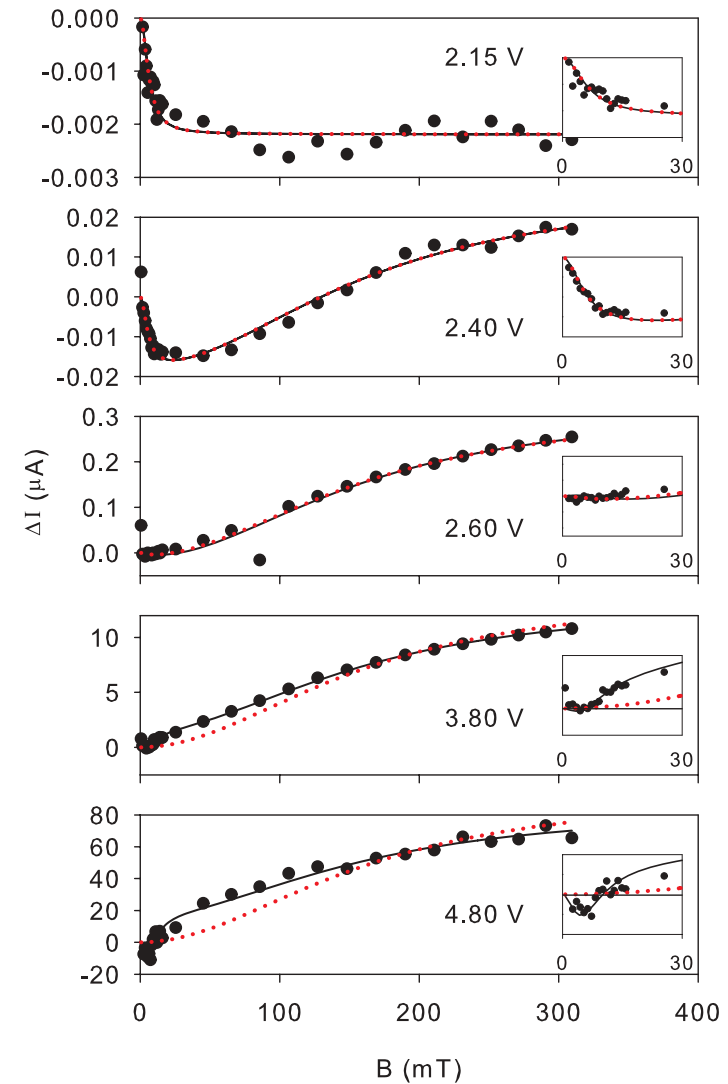

FIG. 3. (Color online) The absolute change in current for a 15-nm $\mathrm{Alq}_{3}$ OLED as a function of magnetic field for several different drive voltages. The solid line is the fit using the trapping, dissociation and interaction components [see Eq. (1)] and the dotted line is the fit using just the trapping and interaction terms. At low voltages, these two fits overlap perfectly. The inset for each graph is the detail of the low-field region from 0 to $30 \mathrm{mT}$.

taking the exciton dissociation component into account (see Fig. 4). The reason for the appearance of exciton dissociation at higher drive voltages can be understood from the fact that exciton formation in TPD/Alq ${ }_{3}$ devices is known to occur at the interface between the TPD and $\mathrm{Alq}_{3}$ (see Ref. 57) due to the fact that the TPD acts as an electron blocking layer. However, as the drive current and exciton concentration increases, the width of the recombination zone must increase until it is sufficiently wide for some of the excitons to be in the vicinity of the cathode and hence liable to dissociate.

Figure 5 shows the magnitude of the triplet-polaron interaction component obtained from the fits, $a_{i}$, plotted against the light output from the device, as we discussed in our earlier work this is a direct measure of the singlet concentration. It can be seen that even for the thin devices, which exhibit a negative OMR at low voltage, the magnitude of the triplet-polaron interaction component scales linearly with light output for all devices over the complete range of operating conditions.

For the exciton trapping and dissociation components, the fitting does not provide unique values for each of these prefactors as it is the difference between them that controls the quality of the fit. In Fig. 6, we have therefore plotted $\left(a_{t}+a_{d}\right)$ against light output. It can be seen that for the 30-nm thick device the data approximately falls on a straight 

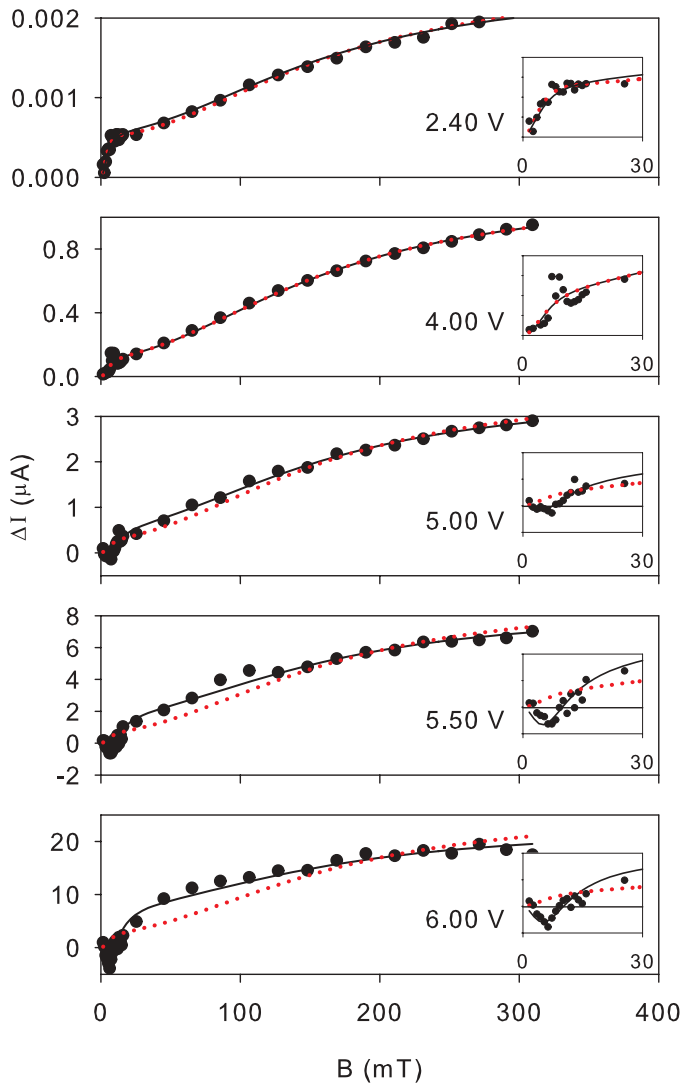

FIG. 4. (Color online) The absolute change in current for a 20-nm $\mathrm{Alq}_{3} \mathrm{OLED}$ as a function of magnetic field for several different drive voltages. The solid line is the fit using the trapping, dissociation and interaction components [see Eq. (1)] and the dotted line is the fit using just the trapping and interaction terms. At low voltages, these two fits overlap perfectly. The inset for each graph is the detail of the low-field region from 0 to $30 \mathrm{mT}$.

line of slope 1 . These data overlap perfectly with the thicker devices presented in Ref. 39. As the thickness is reduced the data for each device, whilst still linear and with a slope one, falls below those of the thicker devices (i.e., a smaller constant of proportionality). These data also cover a smaller range of exciton concentration as the prefactors are initially negative (dissociation dominated), whilst the logarithmic plot only shows the positive (trapping dominated) data.

In our previous work on thick $\mathrm{Alq}_{3}$ devices, ${ }^{39}$ we showed that the dissociation term was found to be proportional to light output over six decades. We have therefore refitted the data and used this observation as a constraint. Equation (2) shows the equation used for this fit where in place of the arbitrary prefactor $a_{t}$ for the trapping component we have used $c L$, where $L$ is the light intensity, in nano-Watts, obtained from that device under the given drive conditions. The value of $c$ chosen for the fitting was $10 \mathrm{~A} / \mathrm{nW}$, which corresponds to the straight line on Fig. 6:

$$
f(B)=c L \frac{B^{2}}{\left(B^{2}+B_{t}^{2}\right)}+a_{d} \frac{B^{2}}{\left(B^{2}+B_{d}^{2}\right)}+a_{i} \frac{B^{2}}{\left(B^{2}+B_{i}^{2}\right)} .
$$

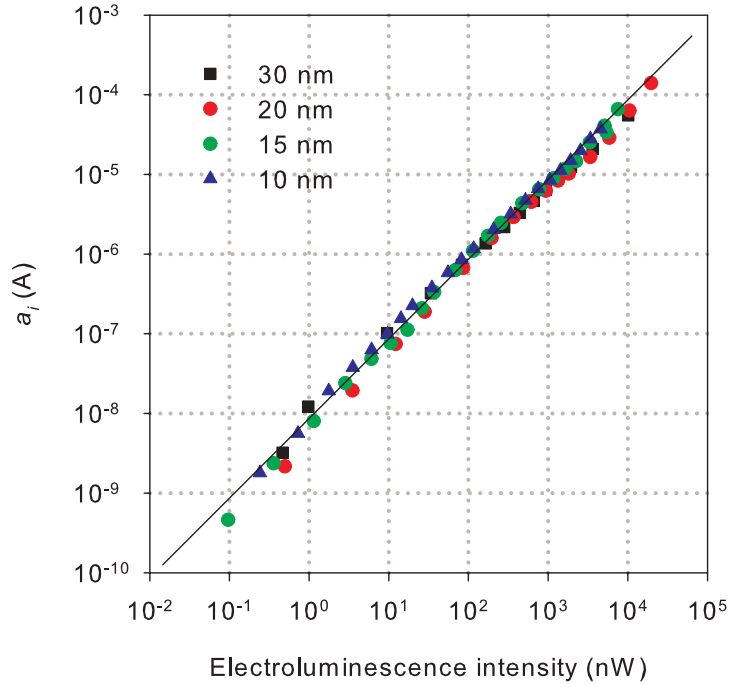

FIG. 5. (Color online) The prefactor for the interaction component of the fit plotted against light output. The straight line is of slope 1 .

Using this approach, the magnitude of the dissociation term was fixed for each data set but we allowed the saturation field to vary as in the previous fitting. This approach therefore allowed us to obtain a consistent set of values for the dissociation prefactor. The quality of these fits was excellent and the full data set and fits are given in Supplementary Material. ${ }^{59}$ Figure 7(a) shows the plot of magnitude of the prefactors for the dissociation term against light output, the straight line is the prefactor for the trapping term calculated from the light output. For the thinnest devices, at low light levels, the magnitude of the dissociation component is significantly greater than that of the trapping component and this accounts for the negative OMR, which dominates in this region. However, once the light output is greater than $10 \mathrm{nW}$, the magnitude of the dissociation and trapping components become roughly comparable. This can be seen more clearly in Fig. 7(b) where we plot the

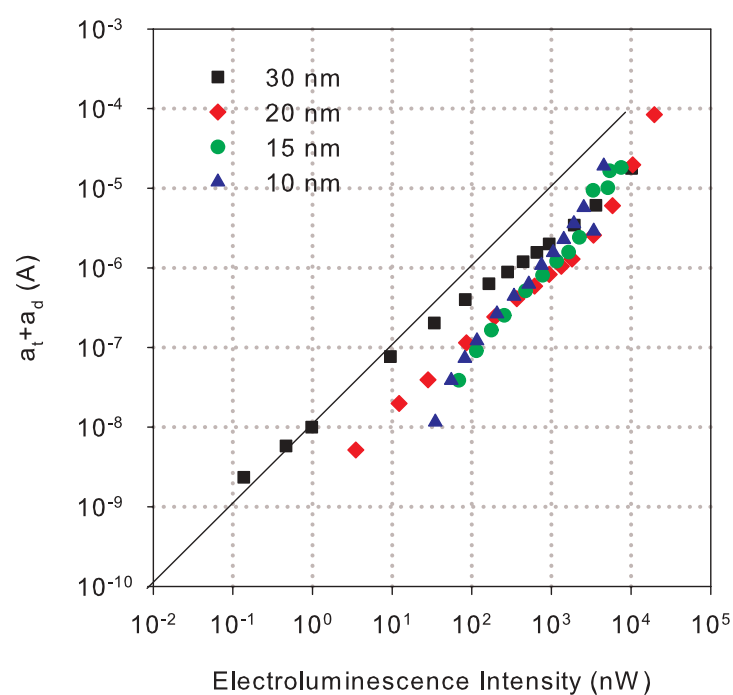

FIG. 6. (Color online) The sum of the prefactors for the dissociation and trapping components of the fit plotted against light output. The straight line is of slope 1 . 

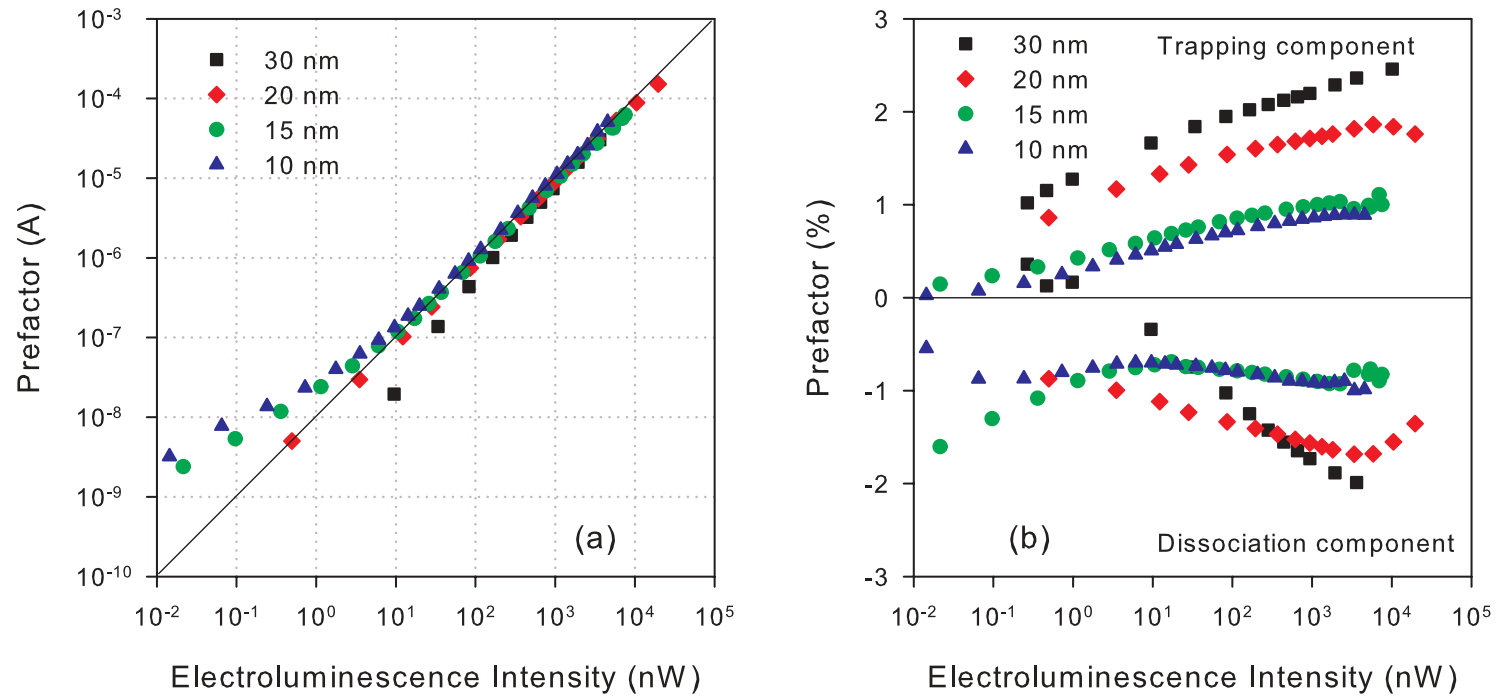

FIG. 7. (Color online) The prefactors for the dissociation and trapping components obtained from Eq. (2) plotted against light output. (a) As the absolute change in current (the trapping component is the solid line) and (b) as the percentage change in current.

same data as a percentage change in current against light output. The positive trapping component, calculated from $c L$ in Eq. (2), can be seen to increase steadily with increasing light output for all devices. Despite the fact that this is calculated directly from the light output measured during the OMR experiment, this rise is due to the fact that the light emission is not perfectly linear with current density but falls slightly as the current density increases. For the two thinnest devices, the percentage change in the dissociation component is found to be approximately constant as the light emission increases. For the thicker devices, however, the magnitude of the dissociation component increases with light emission. This is to be expected as the recombination zone for electrons and holes is expected to be close to the TPD/Alq ${ }_{3}$ interface and so at low exciton concentrations there should not be many excitons near the cathode at low operating voltages. However, as the operating voltage is increased, the recombination zone would be expected to widen and hence the possibility of dissociation at the cathode will increase. Figure 8 shows the prefactor for the interaction term from this analysis plotted as both the absolute value and as the percentage change in current against light output. For this component, it can be seen that there is an initial rise in the prefactor followed be a decrease. This is similar to the OMR response that has been seen by other groups who have plotted the maximum OMR against current.

Given that we suggest that the negative OMR is due to exciton dissociation at the cathode, then physically stopping the excitons in the $\mathrm{Alq}_{3}$ from reaching the cathode should remove the negative process in the OMR. We therefore produced a series of devices with an exciton blocking layer, which consisted of $20 \mathrm{~nm}$ of 2,9-dimethyl-4,7-diphenyl1,10-phenanthroline (BCP), between the $\mathrm{Alq}_{3}$ layer and the
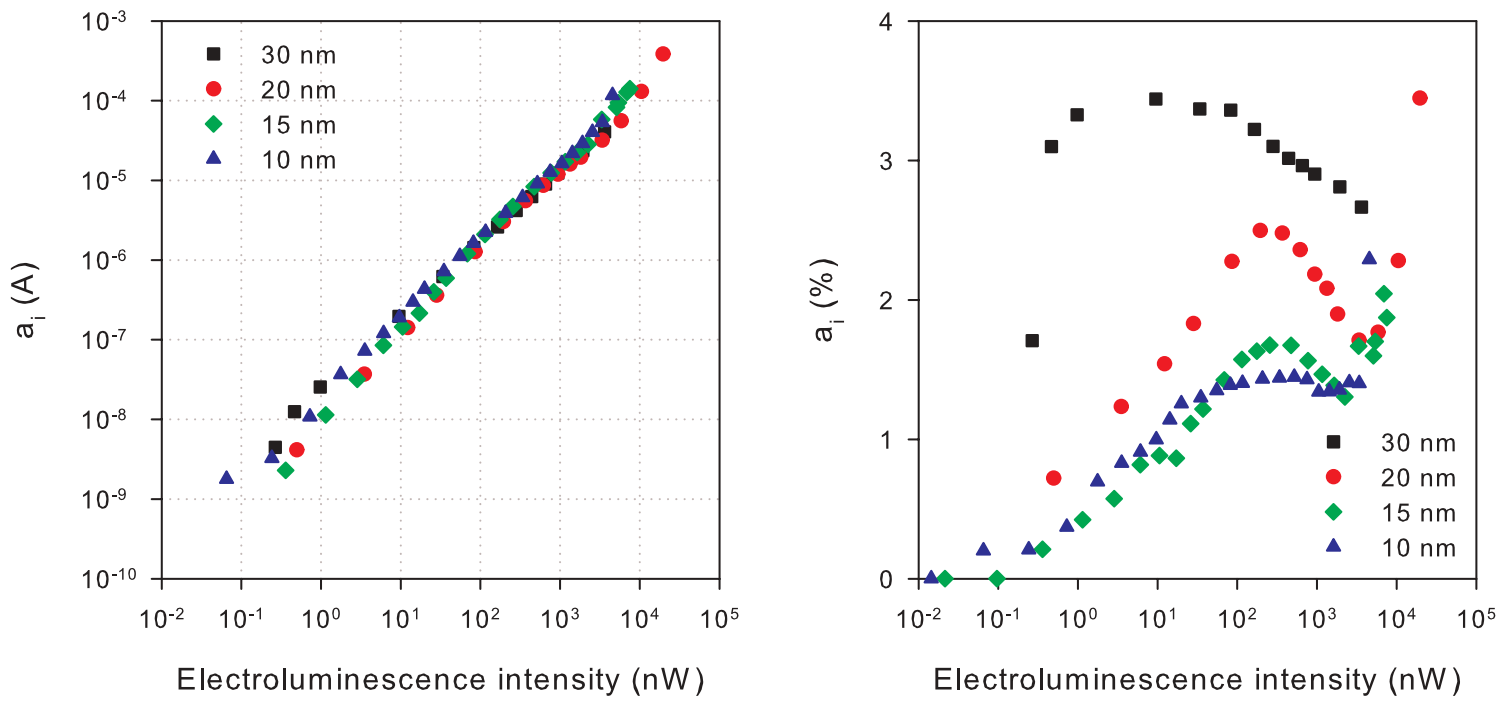

FIG. 8. (Color online) The prefactors for the interaction component obtained from Eq. (2) plotted against light output. (a) As the absolute change in current and (b) as the percentage change in current. 


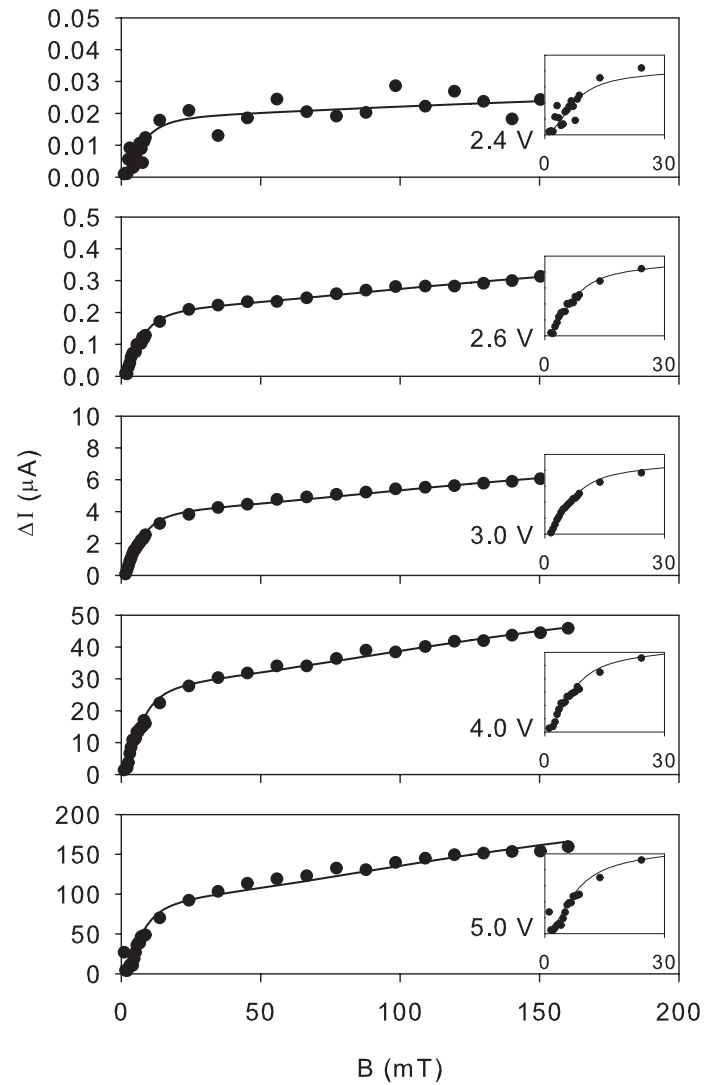

FIG. 9. The absolute change in current for a 15-nm Alq 3 OLED with an exciton blocking layer between the $\mathrm{Alq}_{3}$ and the cathode as a function of magnetic field for several different drive voltages. The solid line is the fit using just the trapping and interaction terms. The inset for each graph is the detail of the low-field region from 0 to $30 \mathrm{mT}$.

$\mathrm{LiF} / \mathrm{Al}$ cathode. These devices were fabricated with $\mathrm{Alq}_{3}$ layer thicknesses of 90, 70, 50, 30, and $15 \mathrm{~nm}$. In none of these devices was there any evidence of negative OMR and in all cases they could be fitted with just the positive exciton-polaron trapping and interaction terms. Figure 9 shows the OMR curves for the device with $15 \mathrm{~nm}$ of $\mathrm{Alq}_{3}$ and a $20 \mathrm{~nm} \mathrm{BCP}$ exciton blocking layer. Without the exciton blocking layer, a $15 \mathrm{~nm} \mathrm{Alq} 3$ device (see Fig. 3) shows clear negative OMR at $2.4 \mathrm{~V}$ and even at $5 \mathrm{~V}$ the OMR curve is dominated by the polaron interaction component as the trapping and dissociation components are approximately equal in magnitude, which results in the small negative peaks in the OMR seen in Figs. 2-4. The complete removal of the negative OMR in the presence of an exciton blocking layer and the fact that this device can be fitted just using the trapping and interaction terms is strong evidence that in these devices the negative OMR is due to exciton dissociation at the cathode interface. For exciton dissociation to occur in any device structure, only the presence of an interface or impurity is required where the dissociation is energetically favorable. Therefore, in other systems, it may be possible to obtain exciton dissociation at a heterojunction within the device rather than at an electrode interface. This is naturally the condition that is required for efficient organic photovoltaic operation. The presence of negative OMR may therefore be indicative of exciton dissociation in the device and could therefore be a useful tool in understanding the internal processes operating in a device.

\section{CONCLUSIONS}

In conclusion, we have shown that it is possible to model both the positive and negative organic magnetoresistance of an $\mathrm{Alq}_{3}$ based OLED using the triplet-polaron interaction model coupled with triplet dissociation at the cathode. More importantly, this work shows that the perturbation of the device current by a magnetic field allows one to probe a number of the mechanisms that are responsible for the current in OLEDs. This technique may therefore provide a new insight into device operation and allows one to see the effect of polaron trapping at triplets, triplet-polaron interaction, and triplet dissociation all in a single working device. This approach opens up a new means of studying current transport in organic devices.
${ }^{1}$ V. Ern and R. E. Merrifield, Phys. Rev. Lett. 21, 609 (1968),

${ }^{2}$ R. C. Johnson and R. E. Merrifield, Phys. Rev. B 1, 896 (1970).

${ }^{3}$ R. P. Groff, A. Suna, P. Avakian, and R. E. Merrifield, Phys. Rev. B 9, 2655 (1974).

${ }^{4}$ C. W. Tang and S. A. VanSlyke, Appl. Phys. Lett. 51, 913 (1987).

${ }^{5}$ J. Kalinowski, J. Szmytkowski, and W. Stampor, Chem. Phys. Lett. 378, 380 (2003)

${ }^{6}$ J. Kalinowski, M. Cocchi, D. Virgili, P. Di Marco, and V. Fattori, Chem. Phys. Lett. 380, 710 (2003).

${ }^{7}$ A. H. Davis and K. Bussmann, J. Vac. Sci. Technol. A 22, 1885 (2004).

${ }^{8}$ T. L. Francis, O. Mermer, G. Veeraraghavan, and M. Wohlgenannt, New J. Phys. 6, 185 (2004).

${ }^{9}$ O. Mermer, G. Veeraraghavan, T. L. Francis, and M. Wohlgenannt, Solid State Commun. 134, 631 (2005).

${ }^{10}$ O. Mermer, G. Veeraraghavan, T. L. Francis, Y. Sheng, D. T. Nguyen, M. Wohlgenannt, A. Kohler, M. K. Al-Suti, and M. S. Khan, Phys. Rev. B 72, 205202 (2005).
${ }^{11}$ Y. Sheng, T. D. Nguyen, G. Veeraraghavan, O. Mermer, M. Wohlgenannt, S. Qiu, and U. Scherf, Phys. Rev. B 74, 045213 (2006).

${ }^{12}$ H. Odaka, Y. Okimoto, T. Yamada, H. Okamoto, M. Kawasaki, and Y. Tokura, Appl. Phys. Lett. 88, 123501 (2006).

${ }^{13}$ V. N. Prigodin, J. D. Bergeson, D. M. Lincoln, and A. J. Epstein, Synth. Met. 156, 757 (2006).

${ }^{14}$ Y. Wu and B. Hu, Appl. Phys. Lett. 89, 203510 (2006).

${ }^{15}$ P. Desai, P. Shakya, T. Kreouzis, W. P. Gillin, N. A. Morley, and M. R. J. Gibbs, Phys. Rev. B 75, 094423 (2007).

${ }^{16}$ P. Desai, P. Shakya, T. Kreouzis, and W. P. Gillin, J. Appl. Phys. 102, 073710 (2007).

${ }^{17}$ P. Desai, P. Shakya, T. Kreouzis, and W. P. Gillin, Phys. Rev. B 76, 235202 (2007).

${ }^{18}$ B. Hu and Y. Wu, Nat. Mater. 6, 985 (2007).

${ }^{19}$ F. L. Bloom, W. Wagemans, M. Kemerink, and B. Koopmans, Phys. Rev. Lett. 99, 257201 (2007). 
${ }^{20}$ Y. Sheng, T. D. Nguyen, G. Veeraraghavan, O. Mermer, and M. Wohlgenannt, Phys. Rev. B 75, 035202 (2007).

${ }^{21}$ Y. Wu, Z. Xu, B. Hu, and J. Howe, Phys. Rev. B 75, 035214 (2007).

${ }^{22}$ T. D. Nguyen, Y. Sheng, J. Rybicki, G. Veeraraghavan, and M. Wohlgenannt, J. Mater. Chem. 17, 1995 (2007).

${ }^{23}$ J. D. Bergeson, V. N. Prigodin, D. M. Lincoln, and A. J. Epstein, Phys. Rev. Lett. 100, 067201 (2008).

${ }^{24}$ F. J. Wang, Heinz Bassler, and Z. Valy Vardeny, Phys. Rev. Lett. 101, 236805 (2008).

${ }^{25}$ N. Rolfe, P. Desai, P. Shakya, T. Kreouzis, and W. P. Gillin, J. Appl. Phys. 104, 083703 (2008).

${ }^{26}$ P. Shakya, P. Desai, T. Kreouzis, W. P. Gillin, S. M. Tuladhar, A. M. Ballantyne, and J. Nelson, J. Phys. Condens. Matter 20, 452203 (2008).

${ }^{27}$ P. Shakya, P. Desai, T. Kreouzis, and W. P. Gillin, J. Appl. Phys. 103, 043706 (2008).

${ }^{28}$ P. Shakya, P. Desai, M. Somerton, G. Gannaway, T. Kreouzis, and W. P. Gillin, J. Appl. Phys. 103, 103715 (2008).

${ }^{29}$ T. H. Lee, T. F. Guo, J. C. A. Huang, and T. C. Wen, Appl. Phys. Lett. 92, 153303 (2008).

${ }^{30}$ C. G. Yang, E. Ehrenfreund, F. Wang, T. Drori, and Z. V. Vardeny, Phys. Rev. B 78, 205312 (2008).

${ }^{31}$ T. D. Nguyen, J. Rybicki, Y. Sheng, and M. Wohlgenannt, Phys. Rev. B 77, 035210 (2008).

${ }^{32}$ T. D. Nguyen, Y. Sheng, J. Rybicki, and M. Wohlgenannt, Phys. Rev. B 77, 235209 (2008).

${ }^{33}$ F. L. Bloom, W. Wagemans, and B. Koopmans, J. Appl. Phys. 103, 07F320 (2008).

${ }^{34}$ F. L. Bloom, W. Wagemans, M. Kemerink, and B. Koopmans, Appl. Phys. Lett. 93, 263302 (2008).

${ }^{35}$ P. A. Bobbert, T. D. Nguyen, F. W. A. van Oost, B. Koopmans, and M. Wohlgenannt, Phys. Rev. Lett. 99, 216801 (2007).

${ }^{36}$ P. A. Bobbert, W. Wagemans, F. W. A. van Oost, B. Koopmans, and M. Wohlgenannt, Phys. Rev. Lett. 102, 156604 (2009).

${ }^{37}$ L. Xin, C. Li, F. Li, S. Liu, and B. Hu, Appl. Phys. Lett. 95, 123306 (2009).

${ }^{38}$ N. J. Rolfe, M. Heeney, P. B. Wyatt, A. J. Drew, T. Kreouzis, and W. P. Gillin, Phys. Rev. B 80, 241201 (2009).

${ }^{39}$ W. P. Gillin, S. J. Zhang, N. J. Rolfe, P. Desai, P. Shakya, A. J. Drew, Kreouzis T. Kreouzis, Phys. Rev. B 82, 195208 (2010).
${ }^{40}$ T. D. Nguyen, B. R. Gautam, E. Ehrenfreund, and Z. V. Vardeny, Phys. Rev. Lett. 105, 166804 (2010).

${ }^{41}$ T. D. Nguyen, G. Hukic-Markosian, F. J. Wang, L. Wojcik, X. G. Li, E. Ehrenfreund, and Z. V. Vardeny, Nat. Mater. 9, 345 (2010).

${ }^{42}$ N. J. Rolfe, M. Heeney, P. B. Wyatt, A. J. Drew, T. Kreouzis, and W. P. Gillin, Synth. Met. 161, 608 (2011).

${ }^{43}$ S. Zhang, A. J. Drew, T. Kreouzis, and W. P. Gillin, Synth. Met. 161, 628 (2011).

${ }^{44}$ S. Zhang, J. Y. Song, T. Kreouzis, and W. P. Gillin, J. Appl. Phys. 106, 043511 (2009).

${ }^{45}$ V. M. Agranovich, D. M. Basko, K. Schmidt, G. C. LaRocca, F. Bassani, S. Forrest, K. Leo, and D. Lidzey, Chem. Phys. 272, 159 (2001).

${ }^{46}$ J. Y. Song, N. Stingelin, W. P. Gillin, and T. Kreouzis, Appl. Phys. Lett. 93, 233306 (2008).

${ }^{47}$ J. Y. Song, N. Stingelin, A. J. Drew, T. Kreouzis, and W. P. Gillin, Phys. Rev. B 82, 085205 (2010).

${ }^{48}$ M. Segal, M. A. Baldo, M. K. Lee, J. Shinar, and Z. G. Soos, Phys. Rev. B 71, 245201 (2005).

${ }^{49}$ G. Li, C. H. Kim, P. A. Lane, and J. Shinar, Phys. Rev. B 69, 165311 (2004).

${ }^{50}$ W. Harneit, C. Boehme, S. Schaefer, K. Huebener, K. Fostiropoulos, and K. Lips, Phys. Rev. Lett. 98, 216601 (2007).

${ }^{51}$ S. Schaefer, S. Saremi, K. Fostiropoulos, J. Behrends, K. Lips, and W. Harneit, Phys. Status Solidi B 245, 2120 (2008).

${ }^{52}$ D. R. McCamey, H. A. Seipel, S. Y. Paik, M. J. Walter, N. J. Borys, J. M. Lupton, and C. Boehme, Nat. Mater. 7, 723 (2008).

${ }^{53}$ D. R. McCamey, K. J. van Schooten, W. J. Baker, S. Y. Lee, S. Y. Paik, J. M. Lupton, and C. Boehme, Phys. Rev. Lett. 104, 017601 (2010).

${ }^{54}$ J. Behrends, A. Schnegg, K. Lips, E. A. Thomsen, A. K. Pandey, I. D. W. Samuel, and D. J. Keeble, Phys. Rev. Lett. 105, 176601 (2010).

${ }^{55}$ M. Colle and C. Garditz, Appl. Phys. Lett. 84, 3160 (2004).

${ }^{56}$ M. Colle, C. Garditz, and M. Braun, J. Appl. Phys. 96, 6133 (2004).

${ }^{57}$ M. A. Baldo and S. R. Forrest, Phys. Rev. B 62, 10958 (2000).

${ }^{58}$ Y. Wu and B. Hu, Appl. Phys. Lett. 89, 203510 (2006).

${ }^{59}$ See Supplemental Material at http://link.aps.org/supplemental/ 10.1103/PhysRevB.86.075206 for the full set of OMR curves for each device. 